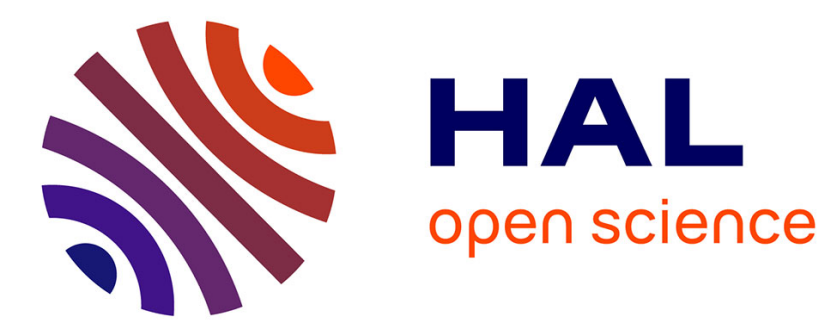

\title{
Efficiency of hyperelastic models for rubber-like materials
}

\author{
Gilles Marckmann, Erwan Verron
}

\section{To cite this version:}

Gilles Marckmann, Erwan Verron. Efficiency of hyperelastic models for rubber-like materials. Fourth European Conference on Constitutive Models for Rubber (ECCMR 2005), Jun 2005, Stockholm, Sweden. pp.375-380. hal-01004682

\section{HAL Id: hal-01004682 \\ https://hal.science/hal-01004682}

Submitted on 7 Oct 2016

HAL is a multi-disciplinary open access archive for the deposit and dissemination of scientific research documents, whether they are published or not. The documents may come from teaching and research institutions in France or abroad, or from public or private research centers.
L'archive ouverte pluridisciplinaire HAL, est destinée au dépôt et à la diffusion de documents scientifiques de niveau recherche, publiés ou non, émanant des établissements d'enseignement et de recherche français ou étrangers, des laboratoires publics ou privés. 


\title{
Efficiency of hyperelastic models for rubber-like materials
}

\author{
G. Marckmann \& E. Verron \\ GeM, École Centrale de Nantes, BP 92101, 44321 Nantes cedex 3, France
}

\begin{abstract}
This paper focuses on the modeling of rubber-like material behaviour under several modes of deformation using hyperelastic constitutive equations. A procedure based on genetic algorithms coupled to classical optimisation methods is proposed to identify the parameters of the models upon experimental data given in the literature. This leads to the classification of nineteen models with respect to criteria related to their capability to predict material behaviour.
\end{abstract}

\section{INTRODUCTION}

Hyperelastic models are used to simulate the nonlinear elasticity of rubber materials under static loading conditions or to develop more sophisticated models. Many models have been proposed to describe the behaviour of elastomers, but few studies which evaluate the ability of hyperelastic models to reproduce rubber behaviour for all the modes of deformation can be found in literature. Recently, Seibert and Schöche (2000) compared six different models with their own experimental data. Danger of the series formulations is highlighted by showing bad predictions of biaxial response after uniaxial identification. Boyce and Arruda (2000) confronted five models with data in three different modes of deformation. More recently, Attard and Hunt (2004) used experimental data of seven different authors to demonstrate the efficiency of their model.

The aim of the present paper is to systematically compare nineteen models proposed in the literature in order to classify them with respect to their ability to fit experimental data.

\section{COMPARED MODELS}

Hyperelastic models are classified into three types of formulation, depending on the approaches adopted by their authors for their development.

\subsection{Phenomenological and empirical models}

The first type concerns general mathematical forms such as the Rivlin series. Their parameters are generally difficult to identify and their generalised form can lead to error when these models are used out of their identification range. Such models considered here are:
- the Mooney model ( 1940).

- the Mooney-Rivlin model (1948).

- the Biderman model (1958).

- the Haines-Wilson model (James et al. 1975) justified by Davet (1985) with experimental considerations.

- (he Ogden (1972) model.

These models are mathematical representations of the strain energy function $W$ with no physical or experimental considerations.

\subsection{Approaches in derivatives $\partial W / \partial I_{1}$ and $\partial W / \partial I_{2}$}

Other authors preferred to extract directly the form of the fonction $\partial W / \partial I_{1}$ and $\partial W / \partial I_{2}$ from experimental data:

- Rivlin et Saunders (1951) observed that $\partial W / \partial I_{1}$ is independent on $I_{1}$ et $I_{2}$ and that $\partial W / \partial I_{2}$ does not depend on $I_{1}$,

- Gent and Thomas (1958) proposed an empirical form with only two material parameters,

- Hart-Smith (1966) noted that $\partial W / \partial I_{1}$ is constant for values of $I_{1}$ smaller than 12 , but increases for higher values. He explained this phenomenon by the limit of extensibility of the polymeric chains,

- Valanis et Landel (1967) proposed a form of $W$ in terms of the principal stretches $\lambda_{i}$ with the assumption of separability on $\lambda_{i}$, 
- Gent (1996) used the idea of a limit of chain extensibility and assumed that $I_{1}$ admits a maximum value,

- Yeoh and Fleming (1997) noted that the reduced stresses tends to a constant value independent of $\left(I_{1}-3\right)$ for $\left(I_{1}-3\right)>5$.

\subsection{Physical-based models}

Over the last decades, development of phenomenological models tends to introduce physical considerations. The third type of models are those which are derived from physics of chains networks. Such models are based on statistical methods leading to different macroscopic models depending on the microscopic phenomena accounted for:

- Treloar (1943) used a gaussian statistical distribution to develop the neo-hookean model,

- Kuhn and Grün (1942) used a non-gaussian theory to take into account the limit chain extensibility. They introduced the inverse Langevin function,

- James and Guth (1943) derived the previous model and proposed a model where chains are re-distributed upon the three principal axes of deformation,

- Ishihara (1951) linearized equations of the nongaussian theory and obtained a Rivlin series where parameters are linked. Thus, he introduced $I_{2}$ into a physical based model (confirmed by Wang and Guth (1952)).

The deviation in experimental data of the ideal chain models is classically imputed to the so-called phantom assumption which does not account for chains entanglement and chains can pass through each other. Authors introduced the idea of entanglement constraints or topology conservation constraints and adopt the following form of the strain energy functions:

$$
W=W_{p h}+W_{c}
$$

where $W_{p h}$ is the phantom network part and $W_{c}$ is the constraints or cross-linking part:

- Ball et al. (1981) developed the slip-link model where a first term corresponds to the phantom Gaussian model,

- Kilian et al. (1986) revived an idea of Wang and Guth by taking into account the van der Waals forces. Few years later the model is presented in a potential form (Ambacher et al. 1989),
- Flory et al. (1994) developed a model where junction points of the chains are constrained to move in a restricted neighbourhood due to the presence of other chains. The phantom part of the model is described by the neo-hookean model,

- Arruda and Boyce (1993) proposed a chain model with a distribution of chains in eight directions,

- Heinrich \& Kaliske ( 1997) built a model where chains are constrained by a tube formed by the surrounding chains. This assumption is attributed to the high degree of entanglement of network chains. The model takes the form of the phenomenological model of Ogden with only two terms,

- Kaliske \& Heinrich (1999) replaced the gaussian distribution of the above tube model by the nongaussian approach and introduced an inextensibility parameter,

- Miehe et al. (2004) developed the non-affine micro-sphere model by associating Langevin chain models with the tube-model. The chains are distributed upon discrete directions and the micro-stretches are allowed to fluctuate around the macro-stretches with only one additional parameter.

\section{IDENTIFICATION METHODS}

It is now established that a unique experimental test is unable to characterize a rubber-like material. Moreover, it is difficult to identify model parameters by fitting only one curve corresponding to one type of deformation, especially when the number of these parameters is large and it is not sure that other types of deformation will be reproduced with good agreement. A good example is given in the paper of Seibert and Schöche (2000).

The incompressible assumption constrains the admissible kinematical field in rubber. In the principal axes, this equation allows the possible deformations to be governed by only two independent variables. Therefore a series of biaxial tests is sufficient to fully identify the constitutive models.

\subsection{Experimental data}

In order to investigate the identification of the material parameters, we choose complementary data from classical papers. The first set of data is due to Treloar (1944) and is widely used by other authors. We only focus on the $8 \% \mathrm{~S}$ vulcanized rubber which is known to exhibit highly reversible elastic behaviour and no crystallization on stretching up to $400 \%$. The specimen was pre-stretched with a initial extension of 
$400 \%$ to eliminate the Mullins effect (Mullins 1948). Experimental measures were performed for equibiaxial extension (EQB), traction (T), pure shear (PS) and combined biaxial extension (BE).

The second set of data is due to Kawabata et al. (1981). With an apparatus built for general biaxial extension testing, they obtained data for a square sheet of polyisopren. The specimen were stretched from 1.04 to 3.7 in one direction $\left(\lambda_{1}\right)$ and from 0.52 to 3.1 in the perpendicular direction $\left(\lambda_{2}\right)$.

The two materials used respectively by Treloar and Kawabata et al. are similar. A unique set of material parameters should be able to reproduce these data with good agreement.

\subsection{Identification algorithms}

The problematic of identification makes analytical solutions to coincide with experimental measurements. The measure of the difference $\phi$ is classically defined by the mean square error. A minimization of $\phi$ is generally employed. In most cases the coincidence of data with theoretical responses can only be established on a restrictive set of data points (validity domain).

Among all possible minimization methods, we focus on classical gradient methods and genetic algorithms. The latest have been used for identification problem for few years (Furukawa \& Yagama 1997; Liu et al. 2002; Yoshimoto et al. 2003).

\subsubsection{Gradient methods}

The solution of the minimization problem is often non-unique. Local solutions are generally obtained with classical methods such as conjugate gradient, Newton-like or Levenberg-Marquardt methods. Such iterative methods consiter the derivatives of $\phi$ and the solution depends on an initial point introduced by users. A series of points is build by looking for a descent direction which allows to find a new solution where the value of $\phi$ is lower than the present one.

\subsubsection{Genetic algorithms}

Genetic algorithms (GA) were introduced by Holland ( 1975). Later Michalewicz resumed the state of the art of such methods ( 1996). A genetic algorithm emulates biological evolutionary theories to solve optimization problems. According to the evolutionary theories, only the most fitting elements in a population are likely to survive and transmit their biological heredity to the next generations. This leads to the evolutive convergence of the species through operator such as competition among individuals, natural selection and mutation of the DNA.
The introduction of randomness in the GA makes exploration of the research space independent of the starting point. Thus the GA are likely to obtain a global optimum of the fitness function instead of a local one.

The original GA was based on a similitude between chromosome and binary code. Crossover of a sequence of bits and mutation bits were tempting to preserve the similitude with biology. Binary coding has long been considered as the best one but other codings are possible and some authors recommend a code as close to the space of parameters as possible. Here, we choose the integer coding.

There is no guaranty for convergence of the solution with the use of GA and no conclusion must be settle from a lonely run. Nevertheless, improvement can be observed by increasing the number of individual while convergence is less sensitive to the number of generation if it is not too small.

\subsection{Identification algorithms}

The choice of the identification algorithm is added to our strategy. Models are first identified with genetic algorithms and material parameters are used as initial parameters in the Levenberg-Marquardt method. In case of divergence of the latest method, the mean square method is used. In such a way, the results always take advantages of the genetic algorithms.

\section{CLASSIFICATION}

\subsection{Identification steps}

Both materials considered by Treloar and Kawabata et.al are similar in terms of composition and behaviour. We will try to determine an unique set of parameters can be identified to reproduce the two sets of experimental data. Two identifications steps are proposed here to achieve this aim :

1. parameters are identified on Treloar's data in traction, pure shear, equibiaxial extension and biaxial extension:

(a) if the accuracy is good, paramaters are retained,

(b) if the accuracy is poor, the validity domain is modified:

i. if the model is not able to reproduce strain hardening at large strain, the domain of validity is reduced for uniaxial extension mode $\left(\lambda_{\max }\right)$,

ii. elsewhere, other modes of deformation are progressively eliminated from the identification procedure. Then, the domain of validity $\left(\lambda_{\max }\right)$ for the other modes of deformation is observed on the response curves.

2. parameters identified by the above step are retained to simulate Kawabata biaxial experiments: 
(a) if the accuracy is good enough, the parameters are considered as model parameters for both materials,

(b) elsewhere, new parameters are identified for the Kawabata data:

i. if the accuracy is not good enough, the validity domain of the model for biaxial extension is reduced.

ii. elsewhere, parameters are retained for biaxial mode and the domain of validity $\left(\lambda_{1}\right.$ and $\left.\lambda_{2}\right)$ is then observed on the response curves.

The strategy described above leads to the determination of parameters for all models and of the validity domain for each mode of deformation.

\subsection{Classification}

The classification presented in Tables 1 and 2 is established with the following criteria. The larger is the domain of validity $\left(\lambda_{\max }, \lambda_{1}\right.$ and $\lambda_{2}$ for the different modes of deformation), the upper is the model in the table. Then, the greater is the number of parameters (nop) of the model, lower is the model. For equivalent models, more consideration is given to the one which can represent both sets of data with the same set of parameters. Finally, a subjective criterion is taken into account to decide between different equivalent models and preferences are awarded to physicalbased models (column Phys in Table 2).

Tables 1 gives the limit $\lambda_{\max }$ of the validity domain for $(\mathrm{T})$ traction, (PS) pure shear, (EQB) equibiaxial extension and (BE) biaxial extension for identification on Treloar data. Notations (under) and (over) indicates if stresses are predicted with underestimation or overestimation.

Tables 2 gives limit of the validity domain $\lambda_{\max }$ in both directions $\left(\lambda_{1}\right.$ and $\left.\lambda_{2}\right)$ for Kawabata et al. data. Notations (under) and (over) indicates if stresses are predicted with underestimation or overestimation. Symbols $(=)$ or $(\neq)$ indicate if only one set of parameters is able to reproduce both sets of data.

\subsection{Example}

The following graphs illustrate the performance of the extended tube model. They are obtained with the same set of parameters for both sets of experimental data. The value of these parameters are given with the notations of Kaliske and Heinrich (1999).

\section{CONCLUSIONS}

This paper focuses on hyperelastic models found in the literature and investigates their capability to reproduce the mechanical behaviour under all kinematically admissible modes of deformation.
Table 1. Classification of hyperelastic models: validity domain for Treloar data

\begin{tabular}{|c|c|c|c|c|c|}
\hline & \multirow[b]{2}{*}{ Model name } & \multicolumn{4}{|c|}{$\begin{array}{l}\text { Treloar data } \\
\lambda_{\max }\end{array}$} \\
\hline & & $\mathrm{T}$ & PS & EQB & $\mathrm{BE}$ \\
\hline 1 & extended tube & - & - & - & - \\
\hline 2 & micro-sphere & - & - & - & - \\
\hline 3 & Ogden & - & - & - & - \\
\hline 4 & Haines-Wilson & - & - & - & - \\
\hline 5 & Biderman & - & - & - & - \\
\hline 6 & Hart-Smith & - & - & - & - \\
\hline 7 & 8-chains & - & - & under & under \\
\hline 8 & Gent & - & - & - & - \\
\hline 9 & Yeoh and Fleming & - & - & - & - \\
\hline 10 & van der Waals & - & - & 2.5 & over \\
\hline 11 & 3-chains & - & - & under & under \\
\hline 12 & tube model & 4 & 3.5 & 3 & - \\
\hline 13 & Mooney & 5 & - & 4 & 2 \\
\hline 14 & Ishihara & 5 & - & 4 & 2.25 \\
\hline 15 & Gent and Thomas & 5 & - & 3 & - \\
\hline 16 & Slip-link & 5 & 4 & 2.5 & over \\
\hline 17 & constr. junctions & 5 & 4 & 2.5 & over \\
\hline 18 & neo-hookean & 5 & 2 & 3 & 2.5 \\
\hline 19 & Valanis and Landel & 3.5 & 2.5 & 1.2 & under \\
\hline
\end{tabular}

Table 2. Classification of hyperelastic models: validity domain for Kawabata et al. data; (nop) number of parameters; (Phys) physical-based model;

\begin{tabular}{|c|c|c|c|c|c|c|}
\hline & \multirow[b]{2}{*}{ Model name } & & \multicolumn{2}{|c|}{$\begin{array}{l}\text { Kawabata } \\
\text { data } \\
\quad \lambda_{\max }\end{array}$} & \multirow[b]{2}{*}{ nop } & \multirow[b]{2}{*}{ Phys } \\
\hline & & & $\lambda_{1}$ & $\lambda_{2}$ & & \\
\hline 1 & extended tube & $=$ & - & - & 4 & $\times$ \\
\hline 2 & micro-sphere & $=$ & - & - & 5 & $x$ \\
\hline 3 & Ogden & $\neq$ & - & - & 6 & \\
\hline 4 & Haines-Wilson & $\neq$ & 3.4 & 3 & 6 & \\
\hline 5 & Biderman & $\neq$ & 2.5 & 3 & 4 & \\
\hline 6 & Hart-Smith & $=$ & 1.9 & 1.5 & 3 & \\
\hline 7 & 8-chains & $\neq$ & 1.9 & 1.9 & 2 & $x$ \\
\hline 8 & Gent & $\neq$ & 1.6 & 1.6 & 2 & \\
\hline 9 & Yeoh and Fleming & $\neq$ & 1.6 & 1.6 & 4 & \\
\hline 10 & van der Waals & $=$ & 2.2 & 2.2 & 4 & $x$ \\
\hline 11 & 3-chains & $\neq$ & 1.3 & 1.3 & 2 & $\times$ \\
\hline 12 & tube model & $=$ & - & - & 3 & $\times$ \\
\hline 13 & Mooney & $\neq$ & 2.2 & 2 & 2 & \\
\hline 14 & Ishihara & $\neq$ & 1.9 & 1.9 & 3 & $\times$ \\
\hline 15 & Gent and Thomas & $=$ & 1.6 & 1.6 & 2 & \\
\hline 16 & Slip-link & $\neq$ & 2.5 & 2.5 & 3 & $\times$ \\
\hline 17 & constr. junctions & $\neq$ & 2.2 & 2.2 & 3 & $\times$ \\
\hline 18 & neo-hookean & $=$ & 1.6 & 1.6 & 1 & $\times$ \\
\hline 19 & Valanis and Landel & $\neq$ & 1.3 & 1.3 & 1 & \\
\hline
\end{tabular}




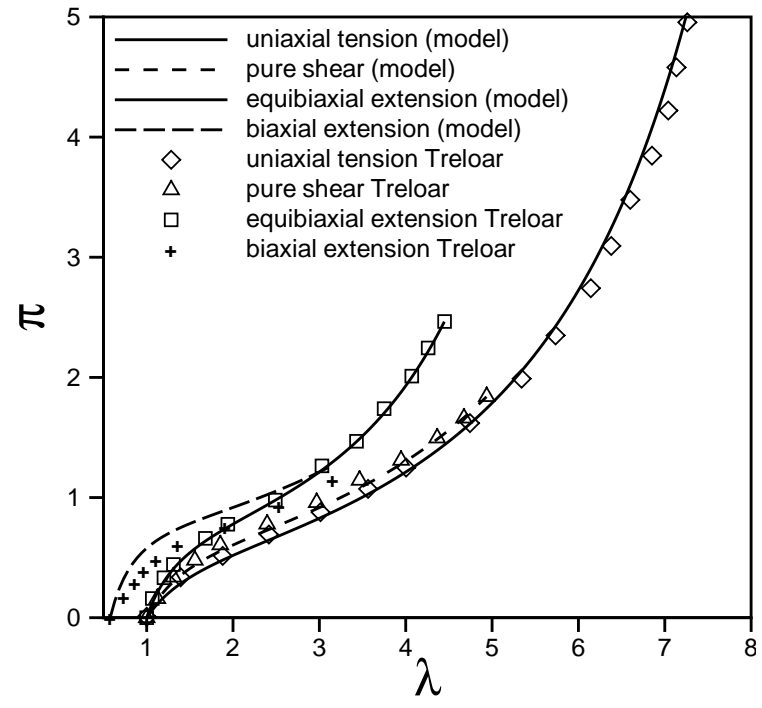

Figure 1. comparison between the extended tube model and experimental data of Treloar: $G_{c}=0.202 ; G_{e}=0.153$; $\beta=0.178 ; \delta=0.0856$

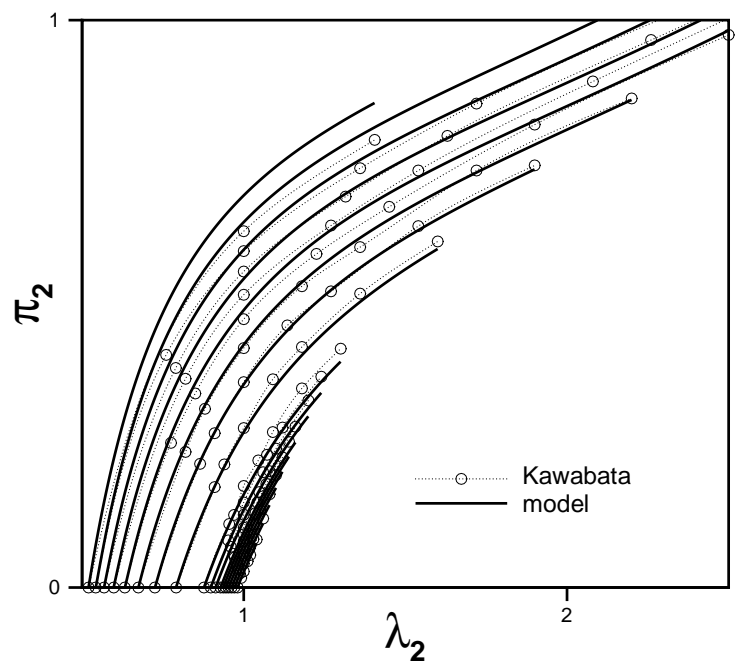

Figure 2. comparison between the extended tube model and experimental data of Kawabata et al.: $G_{c}=0.202 ; G_{e}=$ $0.153 ; \beta=0.178 ; \delta=0.0856$

A methodology is proposed to identify the models with previously published experimental data. An identification procedure has been developed. An original point of this method is the use of genetic algorithms coupled to classical optimisation approaches. The proposed method leads to the identification of both material parameters and of the validity domain of the models.

Finally, a classification of the models is proposed considering the domain of validity for all modes of deformation, the number of parameters and the type of formulation used to derive the models. Depending on the considered domain of deformation, the neohookean model, the Mooney model and the Ogden model can be used respectively for small, moderate or large strain. Nevertheless, the study highlights nonclassically used physical-based models which leads to better agreement with experiments and involves a smaller number of parameters: the extended-tube model and the micro-sphere model.

\section{REFERENCES}

Ambacher, H., H. F. Enderle, H. G. Kilian, \& A. Sauter (1989). Relaxation in permanent networks. Prog. Colloid Polym. Sci. 80, 209-220.

Arruda, E. \& M. C. Boyce (1993). A threedimensional constitutive model for the large stretch behavior of rubber elastic materials. $J$. Mech. Phys. Solids 41(2), 389-412.

Attard, M. M. \& G. W. Hunt (2004). Hyperelastic constitutive modeling under finite strain. Int. J. Solids Struct. 41, 5327-5350.

Ball, R. C., M. Doi, S. F. Edwards, \& M. Warner (1981, August). Elasticity of entangled networks. Polymer 22, 1010-1018.

Biderman, V. L. (1958). Calculations of rubber parts (en russe). Rascheti na Prochnost, 40.

Boyce, M. C. \& E. M. Arruda (2000). Constitutive models of rubber elasticity: a review. Rubber Chem. Technol. 73, 505-523.

Davet, J.-L. (1985, (3ème trimestre). Sur les densités d'énergie en élasticité non linéaire: confrontation de modèles et de travaux expérimentaux. Annales des Ponts et Chaussées.

Flory, P. J. (1994). Network structure and the elasticity properties of vulcanized rubber. Chem. Rev. 35, 51-75.

Furukawa, T. \& G. Yagama (1997). Inelastic constitutive parameter identification using an evolutionary algorithm with continuous individuals. Int. J. Numer. Meth. Engrg. 40, 1071-1090.

Gent, A. N. (1996). A new constitutive relation for rubber. Rubber. Chem. Technol. 69, 59-61.

Gent, A. N. \& A. G. Thomas (1958). Forms of the stored (strain) energy function for vulcanized rubber. J. Polym. Sci. 28, 625-637.

Hart-Smith, L. J. (1966). Elasticity parameters for finite deformations of rubber-like materials. $Z$. angew. Math. Phys. 17, 608-626.

Heinrich, G. \& M. Kaliske (1997). Theoretical and numerical formulation of a molecular based constitutive tube-model of rubber elasticity. Comput. Theo. Polym. Sci. 7(3/4), 227-241.

Holland, J. H. (1975). Adaptation in natural and artificial systems. Ann Arbor: University of Michigan Press. 
Ishihara, A., N. Hashitsume, \& M. Tatibana (1951). Statistical theory of rubber-like elasticity -iv (two dimensional stretching). J. Chem. Phys. 19, 1508-1512.

James, A. G., A. Green, \& G. M. Simpson (1975). Strain energy functions of rubber. i. characterization of gum vulcanizates. J. Appl. Polym. Sci. 19, 2033-2058.

James, H. M. \& E. Guth (1943). Theory of the elastic properties of rubber. J. Chem. Phys. 11, 455-481.

Kaliske, M. \& G. Heinrich (1999). An extended tube-model for rubber elasticity: stastisticalmechanical theory and finite element implantation. Rubb. Chem. Technol. 72, 602-632.

Kawabata, S., M. Matsuda, K. Tei, \& H. Kawai (1981). Experimental survey of the strain energy density function of isoprene rubber vulcanizate. Macromolecules 14, 154-162.

Kilian, H. G., H. F. Enderle, \& K. Unseld (1986). The use of the van der waals model to elucidate universal aspects of structure-property relationships in simply extended dry and swollen rubbers. Colloid Polym. Sci. (264), 866-879.

Kuhn, W. \& F. Grün (1942). Beziehungen zwichen elastischen Konstanten und Dehnungsdoppelbrechung hochelastischer Stoffe. Kolloideitschrift 101, 248-271.

Liu, G., X. Han, \& K. Lam (2002). A combined genetic algorithm and nonlinear least squares methode for material characterization using elastic waves. Comp. Meth. Appl. Mech. Engrg. 191, 1909-1921.

Michalewicz, Z. (1996). Genetic Algorithms + Data Structures = Evolution Programs (Third, revised and extended Edition ed.). Springer. ISBN 3-540-606776-9.

Miehe, C., S. Goktepe, \& F. Lulei (2004). A micromacro approach to rubber-like materials - part i: the non-affine micro-sphere model of rubber elasticity. J. Mech. Phys. Solids 52, 2617-2660.

Mooney, M. (1940). A theory of large elastic deformation. J. Appl. Phys. 11, 582-592.

Mullins, L. (1948). Effect of stretching on the properties of rubber. Rubber Chem. Technol. 21, 281-300.

Ogden, R. W. (1972). Large deformation isotropic elasticity - on the correlation of theory and experiment for incompressible rubberlike solids. Proc. R. Soc. Lond. A 326, 565-584.
Rivlin, R. S. (1948). Some topics in finite elasticity I. Fundamental concepts. Philos. T. Roy. Soc. A 240, 459-490.

Rivlin, R. S. \& D. W. Saunders (1951). Large elastic deformations of isotropic materials - vii. experiments on the deformation of rubber. Philos. T. Roy. Soc. A 243, 251-288.

Seibert, D. J. \& N. Schöche (2000). Direct comparison of some recent rubber elasticity models. Rubber Chem. Technol. 73, 366-384.

Treloar, L. R. G. (1943). The elasticity of a network of long chain molecules (I and II). Trans. Faraday Soc. 39, 36-64 ; 241-246.

Treloar, L. R. G. (1944). Stress-strain data for vulcanised rubber under various types of deformation. Trans. Faraday Soc. 40, 59-70.

Valanis, K. C. \& R. F. Landel (1967). The strain-energy function of a hyperelastic material in terms of the extension ratios. J. Appl. Phys. 38(7), 2997-3002.

Wang, M. C. \& E. Guth (1952). Statistical theory of networks of non-gaussian flexible chains. $J$. Chem. Phys. 20(7), 1144-1157.

Yeoh, O. H. \& P. D. Fleming (1997). A new attempt to reconcile the statistical and phenomenological theories of rubber elasticity. $J$. Polymer Sci. . Part B: Polymer Physics 35, 1919-1931.

Yoshimoto, F., T. Harada, \& Y. Yoshimoto (2003). Data fitting with a spline using a real-coded genetic algorithm. Comp. Aided Design 35, 751760 . 